\title{
Ginsenoside Rg1 inhibits inflammatory responses via modulation of the nuclear factor- $\kappa B$ pathway and inhibition of inflammasome activation in alcoholic hepatitis
}

\author{
JIAJUN LI ${ }^{1 *}$, CHENG YANG $^{1 *}$, SHU ZHANG $^{2}$, SHU LIU $^{1}$, LUOLE ZHAO ${ }^{1}$, \\ HUAN LUO $^{1}$, YATANG CHEN ${ }^{1}$ and WENXIANG HUANG ${ }^{1}$ \\ Departments of ${ }^{1}$ Infectious Diseases and ${ }^{2}$ Hepatobiliary Surgery, \\ The First Affiliated Hospital of Chongqing Medical University, Chongqing 400016, P.R. China
}

Received November 11, 2016; Accepted November 16, 2017

DOI: 10.3892/ijmm.2017.3297

\begin{abstract}
Ginsenoside Rg1 (G-Rg1) is an active ingredient of Panax ginseng, which has previously been reported to attenuate alcohol-induced hepatic damage; however, the underlying mechanisms remain largely unknown. The present study aimed to investigate the protective effects of G-Rg1 on alcohol-induced cell injury in vitro and on a rat model of alcoholic hepatitis in vivo. For the in vitro model, L-O2 cells were incubated with ethanol in the presence or absence of G-Rg1. For the in vivo model, rats were administered ethanol by intragastric injection and were treated with G-Rg1, or dexamethasone as a control. The results indicated that serum biochemical parameters, including alanine aminotransferase, aspartate aminotransferase and total bilirubin, as well as the expression of nuclear factor (NF)- $\kappa \mathrm{B}$ pathway-associated inflammatory cytokines, including interleukin (IL)-6, tumor necrosis factor- $\alpha$ and IL-1 $\beta$, were elevated in response to alcohol; however, they were significantly decreased by G-Rg1 treatment. Furthermore, NF- $\kappa$ B pathway activation was reduced by treatment with G-Rg1. G-Rg1 also decreased oxidative stress by inhibiting cytochrome P450 2E1 expression and reactive oxygen species production, and promoting glutathione peroxidase expression. Furthermore, G-Rg1 inhibited the expression levels of caspase- 3 and -8 , which may be associated with decreased hepatocyte apoptosis. These
\end{abstract}

Correspondence to: Professor Wenxiang Huang or Professor Yatang Chen, Department of Infectious Diseases, The First Affiliated Hospital of Chongqing Medical University, 1 Youyi Road, Yuzhong, Chongqing 400016, P.R. China

E-mail: wenxiang_huang@hotmail.com

E-mail: cyt6535@vip.sina.com

*Contributed equally

Key words: ginsenoside $\mathrm{Rg} 1$, alcoholic liver disease, inflammasome, nuclear factor- $\kappa \mathrm{B}$, anti-inflammatory effects, anti-apoptotic effects data suggested that G-Rg1 may protect hepatocytes against alcohol-induced injury, through preventing excessive inflammation and hepatocellular apoptosis.

\section{Introduction}

Alcohol overconsumption may lead to the occurrence and development of various alcohol-associated liver diseases, including alcoholic steatohepatitis, alcohol-related fibrosis and cirrhosis. Among all hepatic diseases, alcoholic liver disease (ALD) is the second most common cause of morbidity and mortality after viral hepatitis. In addition, the number of ALD cases has been reported to increase annually, and therefore it is considered a critical health problem in China $(1,2)$. Despite the high incidence of alcoholic hepatitis, there is currently no effective treatment available; therefore, novel therapeutic strategies are urgently required. However, achieving this goal has been limited by a poor understanding regarding the pathogenesis underlying alcoholic hepatitis $(3,4)$.

Numerous mechanisms underlying alcohol exposure-induced hepatocellular dysfunction and ALD progression have been proposed, as follows: i) Activation of alcohol dehydrogenase and aldehyde dehydrogenase, which results in overproduction of acetate (5); ii) upregulation of cytochrome P450 2E1 (CYP2E1) expression, which induces alcoholic oxidative stress and hepatotoxicity (6); iii) increased intestinal permeability and serum lipopolysaccharide concentration $(7,8)$; iv) increased production of tumor necrosis factor (TNF)- $\alpha$ and other proinflammatory cytokines, which exacerbates hepatic inflammation (9); v) abnormal lipid metabolism (6); and vi) activation of Kupffer cells and hepatic stellate cells (HSCs), which may lead to collagen synthesis and liver fibrosis (10). However, which factor serves the predominant role remains to be elucidated.

Ginseng is a traditional Chinese medicine that is used as an alternative medicine worldwide, which may affect numerous biological processes, including inflammatory responses, cell proliferation and apoptosis. The key molecules responsible for the pharmacological effects of ginseng are ginsenosides; among them, ginsenoside $\operatorname{Rg} 1$ (G-Rg1) is the most abundant ingredient of Panax ginseng. Previous studies have reported 
that G-Rg1 exerts regulatory effects on reactive oxygen stress, aging, angiogenesis and neuronal differentiation (11-14). In addition, numerous studies have explored the hepatoprotective effects of G-Rg1, and have indicated that G-Rg1 may inhibit the production of proinflammatory factors, including TNF- $\alpha$ (15), and substantially delay the progression of liver fibrosis (16). Recently, G-Rg1 was revealed to promote glucocorticoid (GC) receptor-mediated suppression of nuclear factor (NF)- $\kappa \mathrm{B}$ activation, in order to exert its anti-inflammatory properties (17), and to protect against hepatic ischemia/reperfusion injury $(18,19)$, carbon tetrachloride $\left(\mathrm{CCl}_{4}\right)$-induced liver injury (20) and hepatic fibrosis $(21,22)$. Similarly, our previous study demonstrated that G-Rg1 significantly reduced liver damage in a murine model of acute liver failure (ALF) via the suppression of NF- $\kappa \mathrm{B}$ activation and the inhibition of TNF- $\alpha$-induced, caspase-dependent hepatocellular apoptosis (23). Similar to ALF, other hepatic disorders, including ALD, are accompanied by abundant production of inflammatory cytokines (9). To the best of our knowledge, inflammatory cytokine production is orchestrated by two signals, as follows: i) NF- $\kappa \mathrm{B}$ activation, which subsequently promotes the transcription of numerous inflammation-associated genes, including IL-1 $\beta$, IL- 6 and $\mathrm{TNF}-\alpha$, and provides the basis for generation of these cytokines; ii) activation of inflammasomes by diverse stimulators, namely pathogen-associated molecular patterns and damage-associated molecular patterns, which in turn activate the cysteine protease caspase-1 and promote maturation of proinflammatory cytokines (24). Furthermore, excessive inflammation may lead to a marked loss of hepatocytes. Therefore, it may be hypothesized that G-Rg1 serves anti-inflammatory and anti-apoptotic roles in alcoholic hepatitis, which may be beneficial for its treatment.

The present study aimed to assess the potential of G-Rg1 as a novel therapy for the treatment of alcoholic hepatitis by evaluating its hepatoprotective effects on an alcohol-induced L-O2 cell injury model and on a rat model of alcoholic hepatitis. Furthermore, the molecular mechanisms underlying the hepatoprotective effects of G-Rg1 against damage induced by ethanol and its metabolites were investigated in vitro and in vivo. The present data may provide novel evidence regarding the development of preventive and therapeutic strategies for ALD.

\section{Materials and methods}

Reagents. G-Rg1 (purity $\geq 98.55 \%$ ) in powder form was purchased from Jicui Biotechnology Co., Ltd. (Yunnan, China). Dexamethasone (DEX), the nonfluorescent dye dichlorodihydrofluorescein diacetate $\left(\mathrm{H}_{2} \mathrm{DCFDA}\right)$ and 5,5'-dithiobis(2-nitrobenzoic acid) (DTNB) were obtained from Sigma-Aldrich (Merck KGaA, Darmstadt, Germany). Ethanol (40\%,wt/vol) was purchased from Beijing Dingguo Changsheng Biotechnology Co.,Ltd.(Beijing,China). Assay kits for alanine aminotransferase (ALT; C009-3), aspartate aminotransferase (AST; C010-3) and total bilirubin (TBIL; C019-1) were purchased from Nanjing Jiancheng Bioengineering Institute (Nanjing, China). ELISA kits for the detection of IL-1 (SEA563Ra), IL-6 (SEA079Ra) and TNF- $\alpha$ (SEA133Ra) were purchased from USCN Life Sciences, Inc. (Wuhan, China). PrimeScript reverse transcription (RT) Master Mix kit was purchased from Takara Biotechnology Co., Ltd. (Dalian,
China). SYBR-Green Universal quantitative polymerase chain reaction (qPCR) Master Mix kit was purchased from Bio-Rad Laboratories, Inc. (Hercules, CA, USA). Antibodies against NF- $\mathrm{B}$ (13757-1), CYP2E1 (H00001571-A01), caspase-1 (ABP56678), caspase-3 (ABP50018), caspase-8 (ABP50858) and $\beta$-actin (A01011) were purchased from Abcam (Cambridge, UK). ApopTag ${ }^{\circledR}$ Plus Peroxidase In Situ Apoptosis kit was purchased from EMD Millipore (Billerica, MA, USA).

Cells and cell culture. L-O2 cells (Chongqing Bopei Biotechnology Co., Ltd., Chongqing, China) were cultured in Dulbecco's modified Eagle's medium (DMEM; Gibco; Life Technologies, Grand Island, NY, USA) supplemented with $10 \%$ (v/v) fetal bovine serum (Gibco; Life Technologies) and $1 \%$ penicillin/streptomycin $(100 \mathrm{U} / \mathrm{ml})$ at $37^{\circ} \mathrm{C}$ in a humidified $\mathrm{CO}_{2}$ chamber until cells reached $90 \%$ confluence. Subsequently, cells were collected and plated at $6 \times 10^{3}$ cells/ well in 96-well microplates, after which they were divided into the following groups: Control group, which was treated with saline; model group, which was treated with $40 \%$ ethanol for $48 \mathrm{~h}$; and G-Rg1 group, which was pretreated with 0.5 , 1.0 and $1.5 \mathrm{mg} / \mathrm{ml} \mathrm{G}-\mathrm{Rg} 1$ for $2 \mathrm{~h}$, and was then treated with $40 \%$ ethanol for $48 \mathrm{~h}$; each group consisted of triplicate wells. Cells and culture supernatants were collected after treatment.

Animals and treatment. Rats were housed under standard laboratory conditions for 1 week prior to experimentation, and all animals received humane care in compliance with the Guide for the Care and Use of Laboratory Animals published by the National Institutes of Health (NIH Publication No. 85-23, revised in 1996). Rats were maintained under standard conditions $\left(23+2^{\circ} \mathrm{C}, 55+5 \%\right.$ humidity and $12 \mathrm{~h}$ light-dark cycle) in an air-conditioned room. Rats were allowed free access to a standard rodent diet and water. The present study was approved by the Ethics Committee of Chongqing Medical University (Chongqing, China).

A total of 80 female Sprague-Dawley rats (weight, 200-220 g; age, 7-8 weeks) were purchased from the Animal Facility at Chongqing Medical University. The rats were randomly divided into the following four groups ( $n=20 /$ group): Control, model, G-Rg1 and DEX groups. The alcoholic hepatitis model was established by intragastric administration of ethanol $(4 \mathrm{~g} /$ $\mathrm{kg}$ body weight, twice/day) for 12 consecutive weeks $(23,24)$. Following establishment of the animal model, the G-Rg1 and DEX groups were intragastrically administered G-Rg1 (40 mg/ $\mathrm{kg}$ body weight) or DEX (1 mg/kg) once a day for 5 days, respectively. The control group was treated with an equal volume of saline and was not treated with ethanol. After administration of the indicated treatments, all rats were sacrificed, and blood and liver samples were collected for further analysis.

Electron microscopy. After $48 \mathrm{~h}$ of cell culture, cells were treated with trypsin, and adherent cells were washed and resuspended in centrifuge tubes, which were centrifuged at 10,000 x g for $10 \mathrm{~min}$. Subsequently, $2.5 \%$ glutaraldehyde solution was slowly added along the tube wall, and the ultrastructure of the cells was observed by electron microscopy (S-3000N; Hitachi, Tokyo, Japan).

Biochemical parameter assessment. ALT, AST and TBIL activities in cell culture supernatants and rat serum were measured according to the manufacturer's protocols. Collected 
blood samples were placed at $4^{\circ} \mathrm{C}$ for $2 \mathrm{~h}$ and centrifuged at $4,000 \mathrm{x} \mathrm{g}$ for $15 \mathrm{~min}$ at $4^{\circ} \mathrm{C}$ to obtain the serum.

ELISA. ELISA kits specific for IL-1, IL-6 and TNF- $\alpha$ were conducted according to the manufacturer's protocols, and were performed in triplicate for each sample. Cell culture supernatants and serum were used for ELISA.

Reactive oxygen species (ROS) and gluthathione peroxidase (GSH-Px) detection. ROS were measured using a nonfluorescent dye, $\mathrm{H}_{2}$ DCFDA. Briefly, fluorescence measurements were made in 6-well plates $(10,000$ cells/well). Cells in each group were treated for $48 \mathrm{~h}$, and the cells were washed with 1X PBS and treated with $10 \mu \mathrm{M} \mathrm{H}_{2} \mathrm{DCDDA}$ in the dark at $37^{\circ} \mathrm{C}$ for $20 \mathrm{~min}$. Dichlorofluorescein fluorescence in each well was measured at $37^{\circ} \mathrm{C}$ using a Fluoroskan fluorescence plate reader (Thermo Fisher Scientific, Inc., Waltham, MA, USA) at an excitation wavelength of $488 \mathrm{~nm}$ and an emission wavelength of $538 \mathrm{~nm}$.

The GSH-Px content of the cells was measured using DTNB, according to the method described by Sedlak and Lindsay (27). Briefly, the cell supernatants (500 $\mu \mathrm{l})$ were mixed with $2 \mathrm{ml} 0.2 \mathrm{M}$ phosphate buffer and $10 \mu 10.01 \mathrm{M}$ DTNB in methanol. Absorbance of the mixture was measured at $412 \mathrm{~nm}$.

$R T$-qPCR assay. Total RNA was extracted from the liver samples using TRIzol ${ }^{\mathrm{TM}}$ reagent (15596-026; Invitrogen, Carlsbad, CA, USA) and reverse-transcribed into cDNA using a PrimeScript RT master mix kit according to the manufacturer's protocol. RT-qPCR reactions were conducted in a total volume of $20 \mu \mathrm{l}\left[0.5 \mu \mathrm{l}\right.$ primer $(20 \mu \mathrm{mol} / \mathrm{l}), 1 \mu \mathrm{l} \mathrm{cDNA}, 8 \mu \mathrm{l} \mathrm{H}_{2} \mathrm{O}$ and $10 \mu 12 X$ SYBR-Green qPCR mix] with SYBR-Green Universal qPCR master mix on an ABI 7500 thermocycler (Applied Biosystems; Thermo Fisher Scientific, Inc.). The thermal cycling conditions were as follows: $94^{\circ} \mathrm{C}$ for $2 \mathrm{~min}$, followed by 35 cycles at $94^{\circ} \mathrm{C}$ for $30 \mathrm{sec}, 55^{\circ} \mathrm{C}$ for $30 \mathrm{sec}$ and $72^{\circ} \mathrm{C}$ for $30 \mathrm{sec}$, and a final extension step at $72^{\circ} \mathrm{C} 10 \mathrm{~min}$. The relative expression values were normalized to those of GAPDH, and mRNA fold changes were determined using the $2^{-\Delta \Delta \mathrm{Cq}}$ method (28). Primers used for PCR were as follows: GAPDH, forward 5'-acagcaacagggtggtggac-3', reverse 5'-tttgagggtgcagcgaactt-3'; NF- $\mathrm{B}$, forward 5'-aat ttggcttcctttcttggct-3', reverse 5'-ctgcgataccttaatgacagcg-3'.

Western blot analysis. Western blot analysis was performed as described previously (23). Liver tissues were homogenized in RIPA lysis buffer (WB-0071), which was purchased from Beijing Dingguo Changsheng Biotechnology Co., Ltd. . Briefly, total cell lysates were extracted according to a standard protocol, and protein concentration was determined using a bicinchoninic acid assay kit. Subsequently, the samples were subjected to $10 \%$ SDS-PAGE and proteins (50 $\mu \mathrm{g}$ proteins per sample) were transferred onto a polyvinylidene fluoride membrane (EMD Millipore), which was blocked, for $1 \mathrm{~h}$ at room temperature, with 5\% skim milk in Tris-buffered saline containing $0.5 \%$ Tween-20 . Subsequently, the membrane was incubated with primary antibodies (1:1,000 dilution) specific to NF- $\kappa \mathrm{B}, \mathrm{CY}$ 2E1, caspase $-1,-3$ or -8 overnight at $4^{\circ} \mathrm{C}$, and subsequently incubated with horseradish peroxidase (HRP)-conjugated secondary antibodies (1:1,000 dilution; 705-035-003; Jackson Immunoresearch, West Grove, PA, USA) for $2 \mathrm{~h}$ at $37^{\circ} \mathrm{C}$. HRP signals were detected using an electrochemiluminescence detection system (Intron Biotechnology, Inc., Seongnam, South Korea). For semi-quantification, band densities were determined using Bio-Rad Quantity One software (version 4.6.9; Bio-Rad Laboratories, Inc.), and values were normalized to $\beta$-actin.

Immunohistochemistry and histopathology. Liver tissues from the right lobe were fixed in $4 \%$ buffered paraformaldehyde for $24 \mathrm{~h}$ at $4^{\circ} \mathrm{C}$, embedded in paraffin and cut into $4 \mu \mathrm{m}$ sections using a rotary microtome (Leica RM 2135; Meyer Instruments, Houston, TX, USA). Subsequently, the sections underwent hematoxylin and eosin $(\mathrm{H} \& \mathrm{E})$ and immunohistochemical staining. As previously described (23), sections were dewaxed with xylene and rehydrated, and then microwave antigen retrieval was performed at $95^{\circ} \mathrm{C}$ for $15 \mathrm{~min}$ using $0.01 \mathrm{M}$ citric acid buffer (pH 6.0). The sections were then incubated with a $3 \%$ hydrogen peroxide solution (SP KIT-A1; Beijing Dingguo Changsheng Biotechnology Co., Ltd.) for $10 \mathrm{~min}$ at room temperature. Following several washes in PBS (pH 7.4), sections were blocked with $10 \%$ goat serum and then incubated overnight with primary antibodies [caspase-3 (1:50; 19677-1-AP; Proteintech, Chicago, IL, USA), Casp8 (1:500; ab25901; Abcam), NF-кB p65 (1:1500; ab16502; Abcam)] overnight at $4^{\circ} \mathrm{C}$. After several washes with PBS, biotinylated goat anti-rabbit IgG (1:200; IB-0061; Beijing Dingguo Changsheng Biotechnology Co.,Ltd.) was added at $37^{\circ} \mathrm{C}$ for $30 \mathrm{~min}$. Horseradish peroxidase-labeled streptavidin (1:400; IH-0061; Beijing Dingguo Changsheng Biotechnology Co., Ltd.) was added after several washes with PBS, followed by 30 min of incubation at $37^{\circ} \mathrm{C}$. After additional washes with PBS, immunoreactivity was detected with a diaminobenzidine (DAB) staining kit (AR-0611; Beijing Dingguo Changsheng Biotechnology Co., Ltd.), and sections were counterstained with H\&E. To evaluate the histopathological alterations, samples were examined under a light microscope (Olympus BX51, Hamburg, Germany), and an arbitrary scope was viewed under $x$ 40-400 magnifications; the scores from $\geq 3$ fields/liver section were determined to obtain the mean value. The scores of hepatic inflammation were as follows: score 0 , absent; score 1 , small amount of cells present at the junction of the necrotic zone; score 2, normal amount of cells present; score 3, predominantly neutrophils present; score 4, predominantly mononuclear cells present. Fibrosis extent was graded as follows: score 0 , absent; score 1, thin septa present; score 2, thin septa present linking hepatic veins; score 3, broad/ well-developed septa; score 4, cirrhosis. All specimens were scored by 3 pathologists, each one of whom was blinded to the scoring of the others.

Flow cytometry for cell apoptosis detection. Flow cytometric analysis was conducted according to standard methods. Briefly, after culturing for $48 \mathrm{~h}$, isolated L-O2 cells were adjusted to $1 \times 10^{6}$ cells $/ \mathrm{ml}$, suspended in $100 \mu \mathrm{l}$ binding buffer and stained with FITC-conjugated Annexin V and propidium iodide (Annexin V-FITC/PI Apoptosis Detection kit; 40302ES20; Qcbio Science \& Technologies Co., Ltd., Shanghai, China) for $30 \mathrm{~min}$ at room temperature in the dark. The samples were then washed twice in PBS and were fixed in $1 \%$ paraformaldehyde. Data acquisition was performed on a FACSCalibur flow cytometer (BD Biosciences, Franklin Lakes, NJ, USA) in order to determine the percentage of apoptotic cells. 


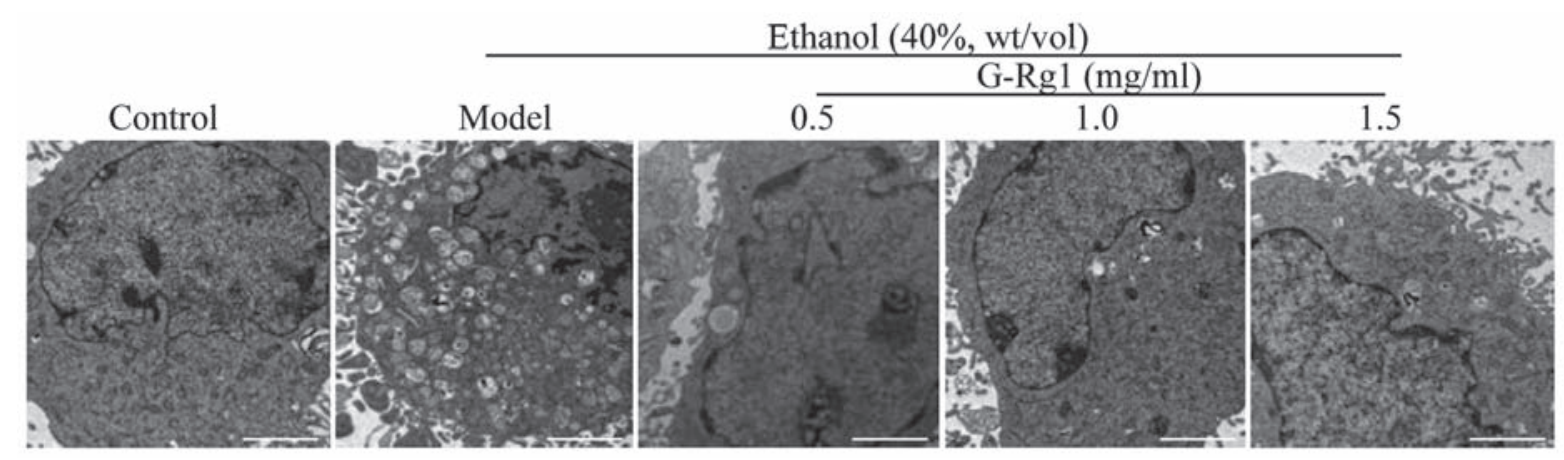

Figure 1. G-Rg1 repairs ethanol-induced damage to the ultrastructure of hepatocytes. L-O2 cells were exposed to $40 \%$ ethanol, and were treated with 0 , $0.5,1.0$ and $1.5 \mathrm{mg} / \mathrm{ml} \mathrm{G-Rg1;} \mathrm{ultrastructure} \mathrm{was} \mathrm{assessed} \mathrm{by} \mathrm{electron} \mathrm{microscopy.} \mathrm{The} \mathrm{normal} \mathrm{control} \mathrm{group} \mathrm{consisted} \mathrm{of} \mathrm{untreated} \mathrm{cells.} \mathrm{Scale} \mathrm{bar,} 2 \mu \mathrm{m}$. Representative data of three independent experiments are shown. G-Rg1, ginsenoside Rg1.

Terminal deoxynucleotidyltransferase-mediated dUTP nick end labeling (TUNEL) staining. A TUNEL assay was performed using the ApopTag ${ }^{\circledR}$ Plus Peroxidase In Situ apoptosis kit (EMD Millipore) according to the manufacturer's protocol, as described previously (23). Liver tissues were used for TUNEL staining.

Statistical analyses. All quantitative values are presented as the mean \pm standard error of the mean and statistical analyses were performed using GraphPad Prism 5.0 software (GraphPad Software, Inc., La Jolla, CA, USA). Statistical significance between two groups was compared using unpaired t-test and multiple comparisons were made using one-way analysis of variance followed by Dunnett or Tukey's post hoc tests. $\mathrm{P}<0.05$ was considered to indicate a statistically significant difference.

\section{Results}

G-Rg1 improves the ultrastructure of ethanol-exposed hepatocytes. Alcohol is able to induce pathological alterations to the subcellular structures of hepatocytes (29). Therefore, the present study aimed to determine whether G-Rg1 could protect hepatocytes from alcohol-induced subcellular damage. L-O2 cells were cultured in DMEM containing $40 \%$ ethanol in combination with $0.5,1.0$ or $1.5 \mathrm{mg} / \mathrm{ml} \mathrm{G}-\mathrm{Rg} 1$, and their ultrastructure was analyzed by electron microscopy. Consistent with the results of Gao et al (17), ethanol-exposed cells exhibited marked alterations in the microstructure, including swelling of cells, loose cell structure and enlarged mitochondria. In addition, no intact endoplasmic reticulum was detectable; however, numerous vacuoles were visible in the cytoplasm. Compared with in the ethanol-treated group, microstructure was markedly improved in cells treated with a low dose of G-Rg1 $(0.5 \mathrm{mg} / \mathrm{ml})$, in which intact mitochondria and fewer vacuoles were detected. When treated with higher doses of G-Rg1 (1.0 and $1.5 \mathrm{mg} / \mathrm{ml}$ ), the microstructure was almost the same as in the control cells (Fig. 1). These results indicated that G-Rg1 may protect organelles against ethanol-induced damage.

$G$-Rg1 restores abnormal levels of biochemical parameters in alcoholic hepatitis. The release of ALT, AST and TBIL is widely used as a biomarker to evaluate hepatocellular damage. To further validate the hepatoprotective activities of G-Rg1, the present study determined the effects of G-Rg1 on the release of these intracytoplasmic proteins in an in vitro ethanol-induced cell injury model and in an in vivo rat hepatitis model. L-O2 cells were cultured in DMEM supplemented with $40 \%$ ethanol, and were treated with G-Rg1 as aforementioned, after which culture supernatants were collected to measure ALT, AST and TBIL. As expected, all three biochemical parameters were elevated in the culture supernatant of ethanol-treated cells, indicating that cellular damage occurred in this group. Conversely, the levels of these parameters were significantly decreased when cells were cotreated with G-Rg1 (Fig. 2A).

To assess the hepatoprotective effects of G-Rg1 in vivo, a rat model of alcoholic hepatitis was generated. Sprague-Dawley rats were intragastrically administered alcohol for 12 consecutive weeks, and were administrated G-Rg1 once a day for a further 5 days. Subsequently, rats were sacrificed, and liver specimens were used to evaluate histological alterations by $\mathrm{H} \& \mathrm{E}$ staining. In addition, serum samples were collected to analyze biochemical parameters. In the liver tissue of normal rats, hepatocytes were polygonal in shape, equal in size and were arranged around the central vein to form lobules. Furthermore, there were no visible lipid droplets in the tissue. Conversely, in rats administered alcohol, hepatocytes exhibited irregular shapes, and lipid vacuoles accumulated in response to alcohol administration, indicating that the alcoholic hepatitis model was successfully established $(25,26,30)$ (Fig. 2B). In accordance with the pathological alterations in liver morphology, serum levels of ALT, AST and TBIL were significantly increased in ethanol-treated rats. However, in rats treated with G-Rg1, these parameters were markedly decreased to levels similar to those in normal rats, and were lower than those in DEX-treated rats (Fig. 2C). Together, these data suggested that G-Rg1 may protect hepatocytes from alcohol-induced injury.

$G$-Rgl decreases the production of proinflammatory cytokines. Alcoholic hepatitis is accompanied with the overproduction of proinflammatory cytokines, which may lead to excessive inflammation and further aggravate hepatocellular damage (9). To evaluate whether G-Rg1 regulates the production of proinflammatory cytokines, ELISA assays were performed on culture supernatants from L-O2 cells treated with ethanol with or without G-Rg1, and on serum samples from rats with alcoholic hepatitis treated with or without G-Rg1.

The results of the present study demonstrated that in the culture supernatants from cells exposed to ethanol, the protein expression levels of IL-6, TNF- $\alpha$ and IL-1 $\beta$ were significantly 
A

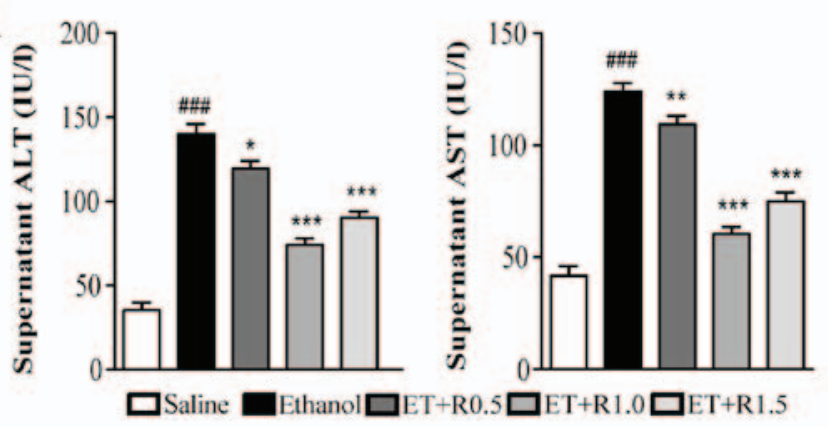

B

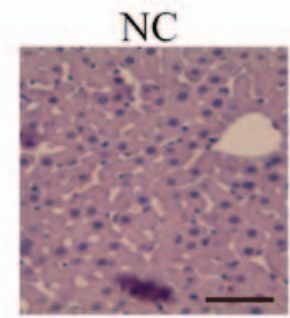

ET (8 weeks)

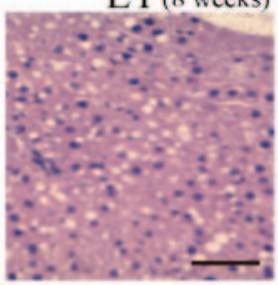

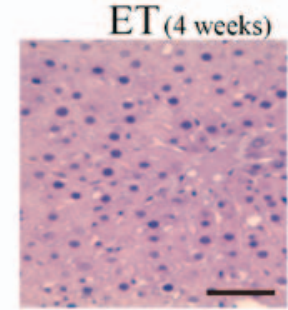

ET(12 weeks)

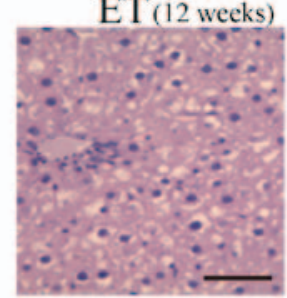

C
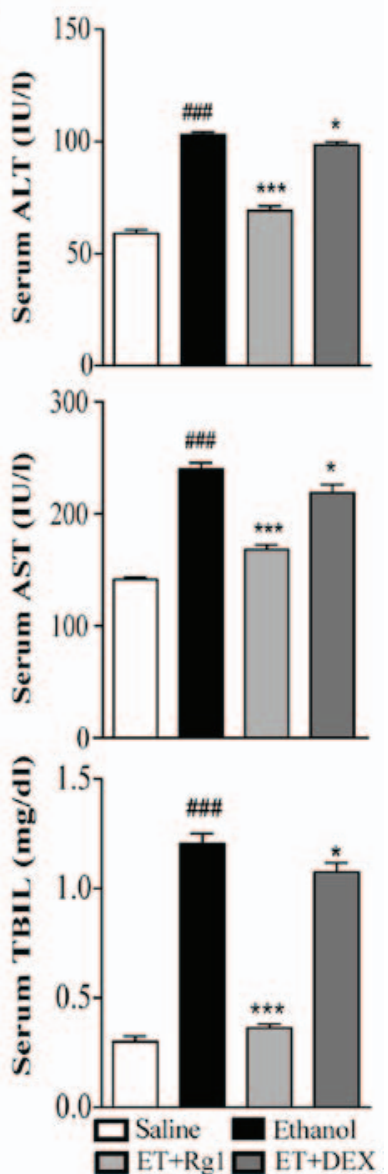

Figure 2. G-Rg1 decreases ethanol-induced biochemical parameter elevation. (A) L-O2 cells were cultured in medium containing ethanol, and were treated with G-Rg1. After a $48 \mathrm{~h}$ culture, AST and ALT in the supernatant were measured using assay kits. (B and C) Rats were intragastrically injected with ethanol for up to 12 weeks to establish the alcoholic liver injury model, and were treated with G-Rg1 or DEX. Following treatment, rats were sacrificed. (B) Liver biopsies were taken to assess ethanol-induced histopathological alterations through hematoxylin and eosin staining. (C) Serum levels of AST, ALT and TBIL were analyzed using assay kits. Scale bar, $50 \mu \mathrm{m}$. Data are presented as the mean \pm standard error of the mean. ${ }^{\# \# "} \mathrm{P}<0.001$ vs. the control group; ${ }^{*} \mathrm{P}<0.05,{ }^{* * *} \mathrm{P}<0.01$ and ${ }^{* * * *} \mathrm{P}<0.001 \mathrm{vs}$. the model/ethanol group. Data from at least two independent experiments are presented. ALT, alanine aminotransferase; AST, aspartate aminotransferase; DEX, dexamethasone; ET, ethanol; G-Rg1, ginsenoside Rg1; NC, normal control; R0.5/1.0/1.5, 0.5/1.0/1.5 mg/ml G-Rg1; TBIL, total bilirubin.
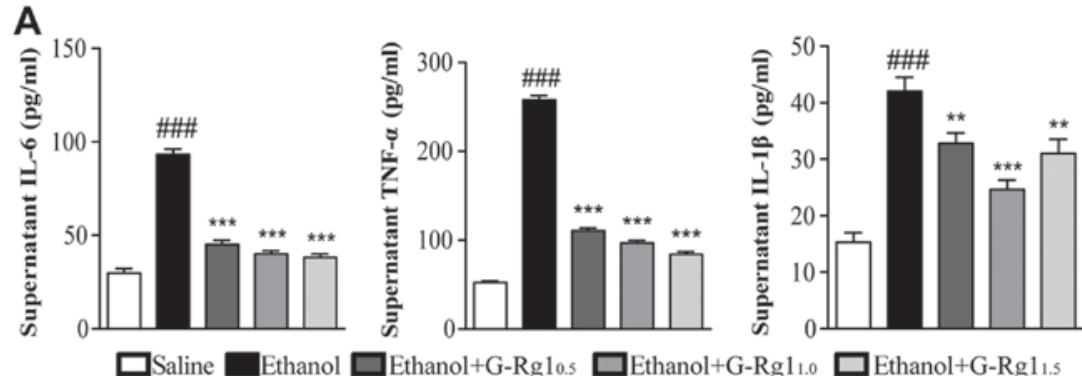

$\square$ Saline $\square$ Ethanol $\square$ Ethanol+G-Rg10.5 $\square$ Ethanol+G-Rg1 1.0 $\square$ Ethanol+G-Rg1 1.5

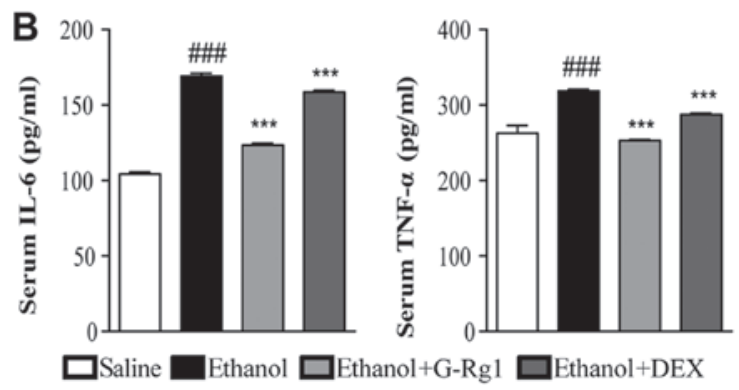

Figure 3. G-Rg1 abrogrates ethanol-induced upregulation of proinflammatory cytokines. (A) IL-6, TNF- $\alpha$ and IL-1 $\beta$ were detected in the culture supernatants of ethanol-exposed L-O2 cells treated with or without G-Rg1 by ELISA. (B) IL- 6 and TNF- $\alpha$ were detected in the serum of untreated, and G-Rg1- or DEX-treated rats with alcoholic hepatitis. Data are presented as the mean \pm standard error of the mean. ${ }^{\# \# \#} \mathrm{P}<0.001 \mathrm{vs.} \mathrm{the} \mathrm{control/saline} \mathrm{group;}{ }^{* * *} \mathrm{P}<0.01$ and ${ }_{* * * *} \mathrm{P}<0.001$ vs. the model/ethanol group. Representative data from three independent experiments are shown. DEX, dexamethasone; G-Rg1, ginsenoside Rg1; IL, interleukin; $\operatorname{Rg} 1 ; \operatorname{Rg} 1_{0.5 / 1.0 / 1.5}, 0.5 / 1.0 / 1.5 \mathrm{mg} / \mathrm{ml} \mathrm{G-Rg1;} \mathrm{TNF-} \alpha$, tumor necrosis factor;- $\alpha$. 


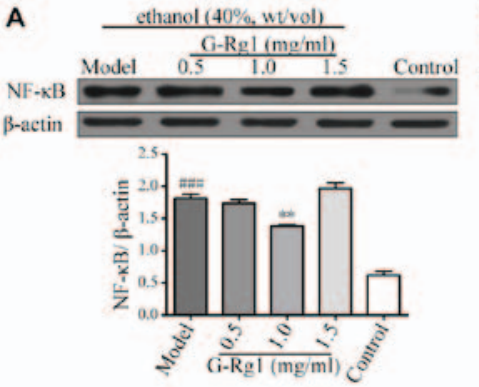

D

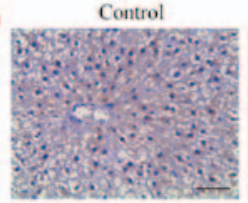

G-Rol
B
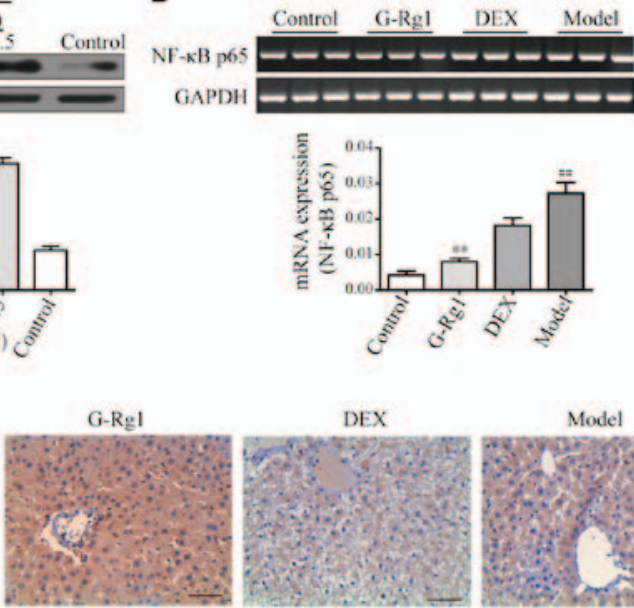

DEX

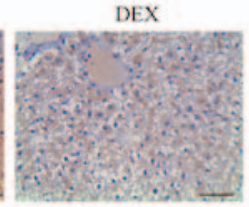

Model

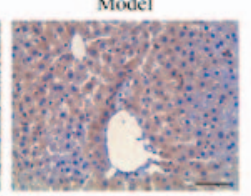

C
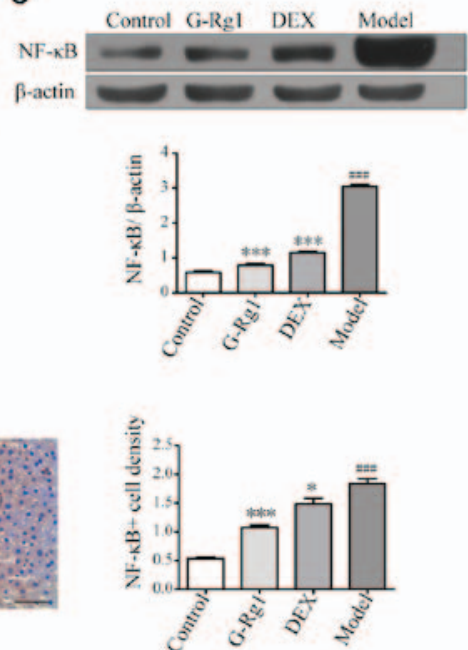

Figure 4. G-Rg1 suppresses activation of the NF- $\mathrm{KB}$ pathway. (A) L-O2 cells were treated with ethanol, ethanol + G-Rg1 (0.5, $1.0 \mathrm{and} 1.5 \mathrm{mg} / \mathrm{ml})$ or saline, and the protein expression levels of NF- $\mathrm{KB}$ were determined by western blot analysis, $\beta$-actin was used as a loading control. (B) Polymerase chain reaction was used to detect NF-KB p65 expression in liver tissues from normal control rats, and rats with alcoholic hepatitis treated with or without G-Rg1 or DEX. GAPDH was used as an internal control. (C) Western blot analysis and (D) immunohistochemical staining for NF- $\kappa$ B expression in the liver tissue of rats. Scale bar, $50 \mu \mathrm{m}$. Data are presented as mean \pm standard error of the mean. ${ }^{\# \#} \mathrm{P}<0.01$ and ${ }^{\# \# \#} \mathrm{P}<0.001$ vs. the control/saline group; ${ }^{*} \mathrm{P}<0.05,{ }^{* * *} \mathrm{P}<0.01$ and ${ }^{* * *} \mathrm{P}<0.001$ vs. the model/ethanol group. Data from at least three independent experiments are shown. DEX, dexamethasone; G-Rg1, ginsenoside Rg1; NF- $\kappa \mathrm{B}$, nuclear factor- $\kappa \mathrm{B}$

A

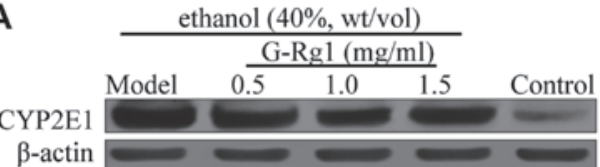

B

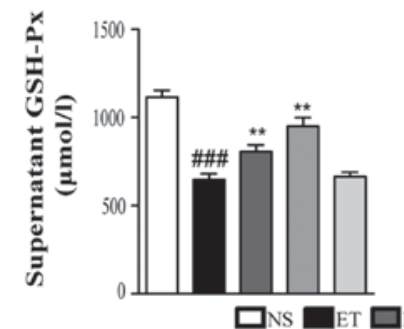

C

D

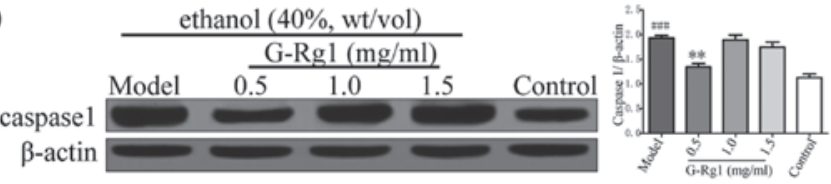

Figure 5. G-Rg1 protects hepatocytes by regulating oxidative stress and inflammasome activation. (A) L-O2 cells were exposed to ethanol and were treated with or without G-Rg1 $(0,0.5,1.0$ and $1.5 \mathrm{mg} / \mathrm{ml})$. CYP2E1 expression was analyzed by western blotting. (B) GSH-Px and (C) ROS were also detected in L-O2 cell supernatants. Data are presented as the mean \pm standard error of the mean.

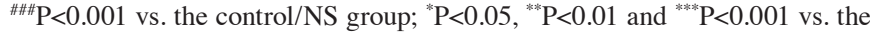
model/ET group. (D) Western blot analysis of caspase-1 expression in L-O2 cells. Three independent experiments with similar results were shown. ET, ethanol; G-Rg1, ginsenoside Rg1; GSH-Px, glutathione peroxidase; NS, normal saline; $\mathrm{R}_{0.5 / 1.0 / 1.5}, 0.5 / 1.0 / 1.5 \mathrm{mg} / \mathrm{ml} \mathrm{G}-\mathrm{Rg} 1$; ROS, reactive oxygen species.

increased compared with in the normal control group. Conversely, when cells were cotreated with G-Rg1, the upregulation of these cytokines was largely abrogated, particularly with regards to IL-6 and TNF- $\alpha$ (Fig. 3A). Similar to the in vitro data, rats with alcoholic hepatitis had higher serum levels of IL-6 and TNF- $\alpha$

compared with in normal rats, and the elevated levels of these two cytokines were partially reduced following treatment with G-Rg1 (Fig. 3B). Therefore, G-Rg1 may modulate the production of proinflammatory cytokines, which may further contribute to the hepatoprotective effects of G-Rg1.

$G-R g 1$ inhibits activation of the $N F-\kappa B$ pathway. The NF- $\kappa \mathrm{B}$ pathway is a key signaling pathway that is involved in the regulation of immune and inflammatory responses, which controls the expression of numerous proinflammatory cytokine genes, including IL-1 $\beta$, IL- 6 and TNF- $\alpha$. To better understand the molecular mechanisms underlying the regulatory effects of G-Rg1 on proinflammatory cytokine production, the present study aimed to determine whether G-Rg1 could inhibit activation of the NF- $\kappa \mathrm{B}$ pathway. L-O2 cells were exposed to ethanol, and were treated with G-Rg1, after which the protein expression levels of NF- $\kappa \mathrm{B}$ were detected by western blot analysis.

The results indicated that $\mathrm{p} 65$ expression was increased in the lysates of ethanol-exposed cells compared with in normal control cells; however, p65 expression was decreased when cells were treated with a moderate dose of G-Rg1 (1.0 mg/ml) (Fig. 4A), thus suggesting that G-Rg1 may negatively regulate $\mathrm{NF}-\kappa \mathrm{B}$ activation. To validate the effects of G-Rg1 on NF- $\mathrm{KB}$ activation in vivo, a rat model of alcoholic hepatitis was established, and rats were treated with G-Rg1 as aforementioned. Initially, the mRNA expression levels of NF- $\mathrm{B}$ p 65 were detected in hepatocytes by RT-qPCR; as shown in Fig. 4B, the mRNA expression levels of $\mathrm{NF}-\kappa \mathrm{B}$ p65 were higher in model rats compared with in normal control rats, whereas they were downregulated by G-Rg1 treatment. Subsequently, the protein expression levels of NF- $\kappa \mathrm{B}$ were determined by western blot analysis. The results demonstrated that p65 was markedly increased in model rats. Conversely, treatment with G-Rg1 resulted in p65 downregulation, to a similar level as that in normal rats (Fig. 4C). To further confirm these results, immunohistochemistry was performed on liver biopsies. Consistent with the western blotting 
A ethanol $(40 \%, w / v o l)$

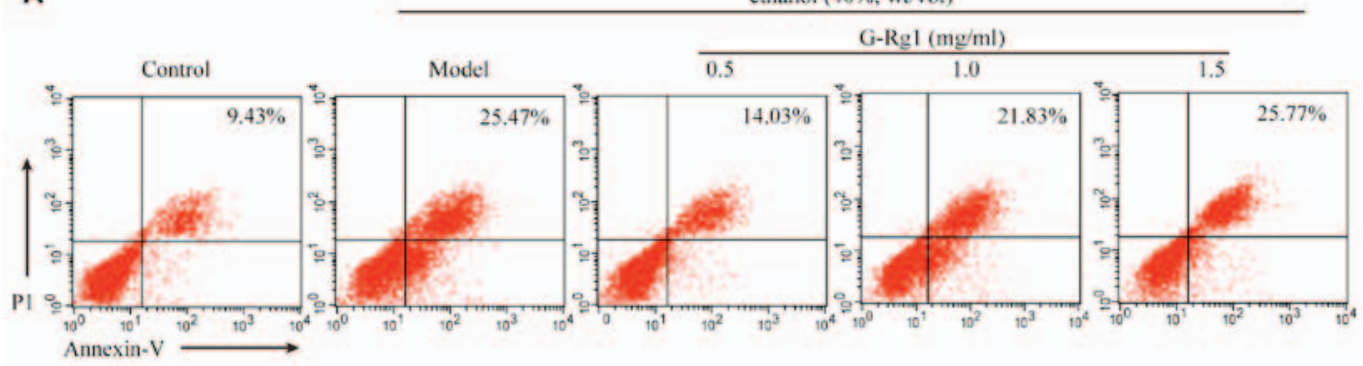

B

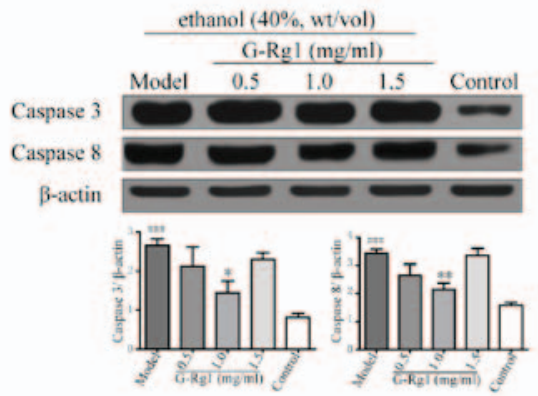

C

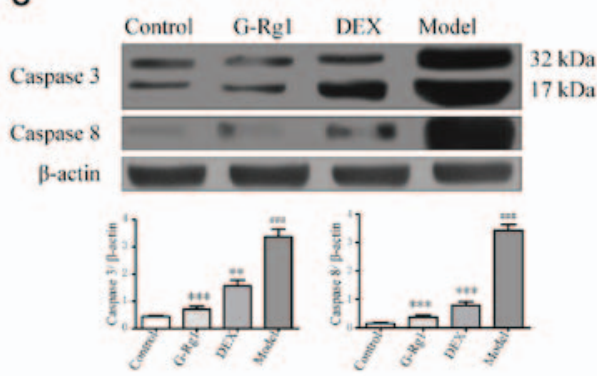

D
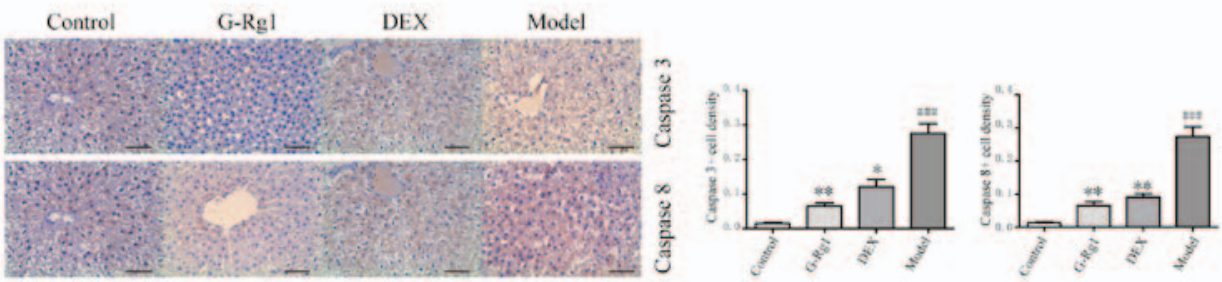

E
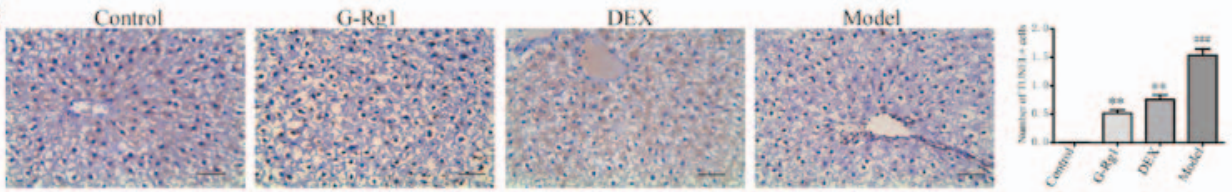

Figure 6. G-Rg1 inhibits hepatocellular apoptosis. L-O2 cells were exposed to ethanol and were treated with or without G-Rg1. (A) Flow cytometric analysis was used to detect apoptosis of L-O2 cells following alcohol exposure. (B) Western blot analysis of caspase-3 and -8 expression in L-O2 cells. (C-E) Rats with alcoholic hepatitis were administered G-Rg1 or DEX treatment, and caspase-3 and -8 expression was detected in liver tissues by (C) western blotting and (D) immunohistochemistry. (E) Apoptosis of hepatocytes was determined by terminal deoxynucleotidyltransferase-mediated dUTP nick end labeling assay. Scale bar, $50 \mu \mathrm{m}$. Data are presented as mean \pm standard error of the mean. ${ }^{\# \# \# *} \mathrm{P}<0.001 \mathrm{vs}$. the control/saline group; ${ }^{*} \mathrm{P}<0.05,{ }^{* *} \mathrm{P}<0.01$ and ${ }^{* * *} \mathrm{P}<0.001 \mathrm{vs}$. the model/ethanol group. Results are from three independent experiments. DEX, dexamethasone; G-Rg1, ginsenoside Rg1

data, p65 expression was increased in liver biopsies from model rats compared with in normal rats, and was markedly decreased by G-Rg1 treatment (Fig. 4D). Taken together, these results suggested that G-Rg1 may inhibit production of proinflammatory cytokines via downregulation of $\mathrm{NF}-\kappa \mathrm{B}$ activation.

G-Rg1 protects hepatocytes by modulating oxidative stress and inflammasome activation. As aforementioned, inflammatory cytokine production is regulated by activation of $\mathrm{NF}-\kappa \mathrm{B}$, which subsequently promotes the transcription of IL-1 $\beta$, IL- 6 and TNF- $\alpha$, and inflammasomes, which in turn activate the cysteine protease caspase-1 and promote the maturation of proinflammatory cytokines (24). During alcoholic hepatitis, metabolism of alcohol increases the levels of CYP2E1, and decreases the levels of antioxidant enzymes, including GSH-Px. As a result, ROS levels are elevated, which finally leads to activation of inflammasomes (31). To elucidate whether the regulatory effects of $\mathrm{G}-\mathrm{Rg} 1$ on proinflammatory cytokine production are associated with inflammasome activation, the expression levels of CYP2E1,
GSH-Px, ROS and caspase-1 were detected in ethanol-exposed cells that were treated with or without G-Rg1.

The results demonstrated that CYP2E1 was upregulated in response to ethanol exposure (Fig. 5A). Conversely, GSH-Px expression was decreased when cells were exposed to ethanol (Fig. 5B). In accordance with these data, cellular ROS wasincreased in ethanol-exposed cells (Fig.5C). However, when cells were treated with a moderate dose of G-Rg1 $(1 \mathrm{mg} / \mathrm{ml})$, CYP2E1 levels were markedly decreased, whereas GSH-Px levels were significantly elevated. Accordingly, abnormal ROS levels were reduced compared with in alcohol-exposed cells (Fig. 5A-C). Notably, expression of caspase-1, which is the essential effector molecule of inflammasomes, was markedly increased in ethanol-exposed cells; however, it was decreased following treatment with G-Rg1 (Fig. 5D). Consistent with these data, the mature form of IL-1 $\beta$, which is dependent on caspase-1 for processing, exhibited higher levels in the culture supernatants of ethanol-treated cells compared with in G-Rg1-treated cells (Fig. 3A). These data suggested that 
G-Rg1 may exert anti-inflammatory effects through inhibition of inflammasome activation.

$G-R g 1$ reduces the apoptosis of hepatocytes. Dysregulated proinflammatory cytokine production may initiate excessive death of hepatocytes, which contributes to the pathogenesis of ALD. Therefore, the present study aimed to determine whether G-Rg1 could promote the survival of hepatocytes in order to exert its hepatoprotective effects. Alcoholic cell injury was induced in L-O2 cells, which were then treated with G-Rg1; cell viability was assessed by flow cytometry, and caspase expression was detected by western blot analysis.

The results indicated that $\sim 25 \%$ of cells underwent apoptosis in the ethanol-treated group, whereas the proportion of apoptotic cells was decreased to $\sim 14 \%$ in cells treated with $0.5 \mathrm{mg} / \mathrm{ml}$ G-Rg1 (Fig. 6A). Corresponding to decreased cell apoptosis, a decline in the protein expression levels of caspase-3 and -8 was detected in G-Rg1-treated cells (Fig. 6B). To verify these results in vivo, the expression levels of caspase-3 and -8 were detected in liver samples from rats with alcoholic hepatitis that were treated with or without G-Rg1. Similar to the in vitro findings, caspase- 3 and -8 expression was increased in model rats, as determined by western blot analysis and immunohistochemistry. Conversely, the levels of caspase- 3 and -8 were markedly decreased in G-Rg1-treated rats (Fig. 6C and D). Consequently, apoptosis of hepatocytes was induced in model rats, whereas it was markedly prevented by G-Rg1 treatment (Fig. 6E). These results indicated that G-Rg1 may alleviate alcoholic hepatitis through suppression of hepatocyte death.

\section{Discussion}

Due to alcohol overconsumption, cases of ALD, including alcohol-induced hepatic steatosis, hepatitis, progressive fibrosis, cirrhosis and hepatocellular carcinoma, are increasing and are considered one of the biggest public health burdens worldwide (32-34). However, at present, there are no strategies that effectively delay or reverse the progression of ALD. Therefore, the identification of an agent able to prevent and treat ALD is urgently required.

G-Rg1, which is the most abundant active ingredient of $P$. ginseng, has been reported to possess hepatoprotective effects in several liver injury models, including ischemia/reperfusion injury, $\mathrm{CCl}_{4}$-induced acute liver failure and hepatic fibrosis (35-37); however, the underlying molecular mechanisms require further elucidation. Consistent with these previous studies, the present study demonstrated that G-Rg1 could protect against alcohol-induced liver damage, as evidenced by improved hepatocyte microstructure, ameliorated hepatic histopathological alterations, and reduced release of biochemical parameters that are hallmarks of hepatocyte damage. Furthermore, the present results suggested that G-Rg1 may protect against ALD through two potential mechanisms: i) Inhibition of excessive inflammatory responses and ii) reduction of hepatocyte apoptosis. Since hepatic inflammation is closely associated with the loss of hepatocytes, and homeostasis of hepatocytes is likely to be maintained by the balance between renewal and death of these cells, the present data thus provided an insight into the hepatoprotective effects of G-Rg1.
Hepatic inflammation, which is associated with the majority of acute and chronic liver disorders, largely determines the outcome of liver damage (38). Mild and short-term inflammation is in favor of repairing tissue damage and the re-establishment of hepatic homeostasis. Conversely, excessive and persistent inflammation may lead to a marked loss of hepatocytes, activation of HSCs and liver fibrosis/cirrhosis $(3,4)$. Therefore, preventing excessive inflammation is the central issue for the treatment of liver diseases. Anti-inflammatory drugs, including GCs, are extensively used to treat inflammatory diseases. G-Rg1 is a type of triterpene saponin, which are homologous to GCs in structure; in the present study, G-Rg1 was revealed to inhibit the production of proinflammatory cytokines. Similar to a previous study (23), G-Rg1 suppressed activation of $\mathrm{NF}-\kappa \mathrm{B}$, which contributed to decreased transcription of inflammation-associated genes. In addition, G-Rg1 modulated the levels of CYP2E1, GSH-Px and ROS, which in turn affected the activation of inflammasomes and the downstream effector molecule caspase-1, and finally regulated the maturation of proinflammatory cytokines. Notably, G-Rg1 was as potent as DEX in suppression of proinflammatory cytokine production and $\mathrm{NF}-\kappa \mathrm{B}$ activation, whereas much fewer side effects were reported by other studies $(39,40)$. Therefore, the present study provided information regarding the molecular mechanisms underlying the anti-inflammatory effects of G-Rg1, and indicated that exploring chemical components from traditional herbs may be a promising direction for the development of novel drugs for the treatment of inflammatory diseases, including ALD.

Although G-Rg1 was able to protect hepatocytes against alcohol-induced injury, it should be noted that besides hepatocytes, the liver is composed of diverse types of resident and circulating immune cells. These cells include macrophages (Kupffer cells), monocytes, neutrophils, dendritic cells, natural killer cells, natural killer $\mathrm{T}$ cells and $\mathrm{T}$ lymphocytes. While cytokines serve a crucial role in initiating and maintaining hepatic inflammation and liver damage, the major source of these cytokines is immune cells (41). Therefore, immune cells should be dominant in determining the outcomes of inflammatory responses in the liver, as well as the progression of hepatic disorders. However, there is currently little information regarding the regulatory effects of G-Rg1 on the functionality and cellular behaviors of immune cells. Further studies to elucidate these effects may help broaden current understanding regarding the hepatoprotective effects of G-Rg1 and could be beneficial for the treatment of liver diseases.

In conclusion, the present study demonstrated that G-Rg1 was capable of protecting the liver from alcohol-induced injury through inhibition of inflammatory responses and hepatocellular apoptosis. These results may help to further elucidate the molecular mechanisms underlying the hepatoprotective effects of G-Rg1 and evaluate the potential of G-Rg1 as a candidate agent for the prevention and treatment of ALD.

\section{Acknowledgements}

The authors would like to thank Dr Zhengyu Shi for helping with the statistical analysis, and Dr Chengwei Liu for providing technical support (both from Department of Infectious Diseases, The First Affliated Hospital of Chongqing Medical 
University, Chongqing, China). The present study was supported by the Chongqing Science Foundation (grant no. KJ130332 to Wenxiang Huang).

\section{References}

1. Chang B,Li B,Huang A, Sun Y, Teng G, Wang X, LiangpunsakulS, Li J and Zou Z: Changes of four common non-infectious liver diseases for the hospitalized patients in Beijing 302 hospital from 2002 to 2013. Alcohol 54: 61-65, 2016.

2. Wang H, Ma L, Yin Q, Zhang X and Zhang C: Prevalence of alcoholic liver disease and its association with socioeconomic status in north-eastern China. Alcohol Clin Exp Res 38: 1035-1041, 2014.

3. Iwaisako K, Brenner DA and Kisseleva T: What's new in liver fibrosis? The origin of myofibroblasts in liver fibrosis. J Gastroenterol Hepatol 27 (Suppl 2): 65-68, 2012.

4. Schattenberg JM, Galle PR and Schuchmann M: Apoptosis in liver disease. Liver Int 26: 904-911, 2006.

5. Zakhari S and Li TK: Determinants of alcohol use and abuse: Impact of quantity and frequency patterns on liver disease. Hepatology 46: 2032-2039, 2007.

6. You M and Crabb DW: Recent advances in alcoholic liver disease II. Minireview: Molecular mechanisms of alcoholic fatty liver. Am J Physiol Gastrointest Liver Physiol 287: G1-G6, 2004

7. Nagata K, Suzuki H and Sakaguchi S: Common pathogenic mechanism in development progression of liver injury caused by non-alcoholic or alcoholic steatohepatitis. J Toxicol Sci 32: 453-468, 2007

8. Enomoto N, Ikejima K, Bradford BU, Rivera CA, Kono H, Goto M, Yamashina S, Schemmer P, Kitamura T, Oide H, et al Role of Kupffer cells and gut-derived endotoxins in alcoholic liver injury. J Gastroenterol Hepatol 15 (Suppl): D20-D25, 2000.

9. Gao B: Cytokines, STATs and liver disease. Cell Mol Immunol 2: 92-100, 2005.

10. Friedman SL: Hepatic stellate cells: Protean, multifunctional, and enigmatic cells of the liver. Physiol Rev 88: 125-172, 2008.

11. Xie XS, Liu HC, Wang FP, Zhang CL, Zuo C, Deng Y and Fan JM: Ginsenoside Rg1 modulation on thrombospondin-1 and vascular endothelial growth factor expression in early renal fibrogenesis in unilateral obstruction. Phytother Res 24: 1581-1587, 2010.

12. Xu L, Chen WF and Wong MS: Ginsenoside Rg1 protects dopaminergic neurons in a rat model of Parkinson's disease through the IGF-I receptor signalling pathway. Br J Pharmacol 158: 738-748, 2009

13. Shen LH and Żhang JT: Ginsenoside Rg1 promotes proliferation of hippocampal progenitor cells. Neurol Res 26: 422-428, 2004.

14. Shi AW, Wang XB, Lu FX, Zhu MM, Kong XQ and Cao KJ: Ginsenoside Rg1 promotes endothelial progenitor cell migration and proliferation. Acta Pharmacol Sin 30: 299-306, 2009.

15. Wu CF, Bi XL, Yang JY, Zhan JY, Dong YX, Wang JH, Wang JM, Zhang R and Li X: Differential effects of ginsenosides on NO and TNF-alpha production by LPS-activated N9 microglia. Int Immunopharmacol 7: 313-320, 2007.

16. Wu F, Zhang SS and Kang GF: Effects of panax notoginseng saponins on the expression of tumor necrosis factor alpha and secretion phospholipase A2 in rats with liver fibrosis. Zhonghua Gan Zang Bing Za Zhi 11: 51-52, 2003 (In Chinese).

17. Gao Y, Chu S, Li J, Li J, Zhang Z, Xia C, Heng Y, Zhang M, Hu J, Wei $\mathrm{G}$, et al: Anti-inflammatory function of ginsenoside Rg1 on alcoholic hepatitis through glucocorticoid receptor related nuclear factor-kappa B pathway. J Ethnopharmacol 173: 231-240, 2015.

18. Tao T, Chen F, Bo L, Xie Q, Yi W, Zou Y, Hu B, Li J and Deng X: Ginsenoside $\operatorname{Rg} 1$ protects mouse liver against ischemia-reperfusion injury through anti-inflammatory and anti-apoptosis properties. J Surg Res 191: 231-238, 2014

19. Zhang XJ, He C, Li P, Su H and Wan JB: Ginsenoside Rg1, a potential $\mathrm{JNK}$ inhibitor, protects against ischemia/reperfusion-induced liver damage. J Funct Foods 15: 580-592, 2015.

20. Xin Y, Wei J, Chunhua M, Danhong Y, Jianguo Z, Zongqi C and Jian-An B: Protective effects of Ginsenoside Rg1 against carbon tetrachloride-induced liver injury in mice through suppression of inflammation. Phytomedicine 23: 583-588, 2016.
21. Geng J, Peng W, Huang Y, Fan H and Li S: Ginsenoside-Rg1 from Panax notoginseng prevents hepatic fibrosis induced by thioacetamide in rats. Eur J Pharmacol 634: 162-169, 2010.

22. Li JP, Gao Y, Chu SF, Zhang Z, Xia CY, Mou Z, Song XY, He WB, Guo XF and Chen NH: Nrf2 pathway activation contributes to anti-fibrosis effects of ginsenoside Rg1 in a rat model of alcoholand $\mathrm{CCl}_{4}$-induced hepatic fibrosis. Acta Pharmacol Sin 35: 1031-1044, 2014.

23. Zhao J, Shi Z, Liu S, Li J and Huang W: Ginsenosides Rg1 from Panax ginseng: A potential therapy for acute liver failure patients? Evid Based Complement Alternat Med 2014: 538059, 2014.

24. Bieghs V and Trautwein C: The innate immune response during liver inflammation and metabolic disease. Trends Immunol 34: 446-452, 2013

25. Tsukamoto H, Reidelberger RD, French SW and Largman C: Long-term cannulation model for blood sampling and intragastric infusion in the rat. Am J Physiol 247: R595-R599, 1984.

26. Tsukamoto H, Horne W, Kamimura S, Niemelä O, Parkkila S, Ylä-Herttuala S and Brittenham GM: Experimental liver cirrhosis induced by alcohol and iron. J Clin Invest 96: 620-630, 1995.

27. Sedlak J and Lindsay RH: Estimation of total, protein-bound, and nonprotein sulfhydryl groups in tissue with Ellman's reagent. Anal Biochem 25: 192-205, 1968.

28. Livak KJ and Schmittgen TD: Analysis of relative gene expression data using real-time quantitative PCR and the 2(-Delta Delta C(T)) Method. Methods 25: 402-408, 2001.

29. Santos Rocha SW, Silva BS, Gomes FO, Soares e Silva AK, Raposo C, Barbosa KP, Torres DO, dos Santos AC and Peixoto CA: Effect of diethylcarbamazine on chronic hepatic inflammation induced by alcohol in C57BL/6 mice. Eur J Pharmacol 689: 194-203, 2012.

30. Singal AK, Kodali S, Vucovich LA, Darley-Usmar V and Schiano TD: Diagnosis and treatment of alcoholic hepatitis: A systematic review. Alcohol Clin Exp Res 40: 1390-1402, 2016.

31. Szabo $G$ and Csak T: Inflammasomes in liver diseases. J Hepatol 57: 642-654, 2012.

32. Walker RK, Cousins VM, Umoh NA, Jeffress MA, Taghipour D, Al-Rubaiee $M$ and Haddad GE: The good, the bad, and the ugly with alcohol use and abuse on the heart. Alcohol Clin Exp Res 37: 1253-1260, 2013.

33. Rehm J, Samokhvalov AV and Shield KD: Global burden of alcoholic liver diseases. J Hepatol 59: 160-168, 2013.

34. Liu J, Wang X, Liu R, Liu Y, Zhang T, Fu H and Hai C: Oleanolic acid co-administration alleviates ethanol-induced hepatic injury via Nrf-2 and ethanol-metabolizing modulating in rats. Chem Biol Interact 221: 88-98, 2014.

35. Zou Y, Tao T, Tian Y, Zhu J, Cao L, Deng X and Li J: Ginsenoside Rg1 improves survival in a murine model of polymicrobial sepsis by suppressing the inflammatory response and apoptosis of lymphocytes. J Surg Res 183: 760-766, 2013.

36. Shen L and Zhang J: Ginsenoside Rg1 increases ischemia-induced cell proliferation and survival in the dentate gyrus of adult gerbils. Neurosci Lett 344: 1-4, 2003.

37. Karikura M, Tanizawa H, Hirata T, Miyase T and Takino Y: Studies on absorption, distribution, excretion and metabolism of ginseng saponins. VIII. Isotope labeling of ginsenoside $\mathrm{Rb} 2$. Chem Pharm Bull (Tokyo) 40: 2458-2460, 1992.

38. Kubes P and Mehal WZ: Sterile inflammation in the liver. Gastroenterology 143: 1158-1172, 2012.

39. Cao YW, Jiang Y, Zhang DY, Wang M, Chen WS, Su H, Wang YT and Wan JB: Protective effects of Penthorum chinense Pursh against chronic ethanol-induced liver injury in mice. J Ethnopharmacol 161: 92-98, 2015.

40. Ding RB, Tian K, Huang LL, He CW, Jiang Y, Wang YT and Wan JB: Herbal medicines for the prevention of alcoholic liver disease: A review. J Ethnopharmacol 144: 457-465, 2012.

41. Brenner C, Galluzzi L, Kepp O and Kroemer G: Decoding cell death signals in liver inflammation. J Hepatol 59: 583-594, 2013.

This work is licensed under a Creative Commons Attribution-NonCommercial-NoDerivatives 4.0 International (CC BY-NC-ND 4.0) License. 